\title{
Evaluating Human Natural Killer Cells Antibody-dependent Cellular Cytotoxicity (ADCC) Using Plate-bound Anti-CD16 Antibodies
}

Weiru Liu ${ }^{1, \$, *}$

\author{
${ }^{1}$ Center for Immunology and Infectious Disease, University of California Davis, Davis, CA, USA \\ \$Present address: Department of Cancer Biology, Perelman School of Medicine, University of \\ Pennsylvania, Philadelphia, PA, USA \\ *For correspondence: wrliu@ucdavis.edu
}

[Abstract] Natural killer (NK) cells are large granular lymphocytes that keep in check the health of neighboring cells through a large array of intrinsically expressed germline-coded receptors. Most importantly, CD16 is a low affinity Fc receptor for IgG that mediates the antibody-dependent cellular cytotoxicity (ADCC) of NK cells, bridging the innate and adaptive immunities. There has been a significant interest in genetically engineering NK cells to enhance its ADCC, with the ultimate goal to produce off-the-shelf NK cell therapy products that can be combined with target-specific monoclonal antibodies to improve clinical outcomes. Previous protocols of ADCC assays use complex cell-based antigen-antibody models, which are both costly and time-consuming. This current protocol is devoid of target cells and uses plate-bound immobilized anti-CD16 antibodies as the trigger. It greatly shortens the experimental time, while faithfully evaluating NK cells ADCC.

\section{Graphic abstract:}

\section{A. Plate coating}

B. NK cell preparation (for fresh NK cells: PBMC isolation; for expanded NK cells: resting)

\section{$\downarrow$}

\section{Incubation/stimulation}

$\downarrow$

\section{Flow cytometric analysis}

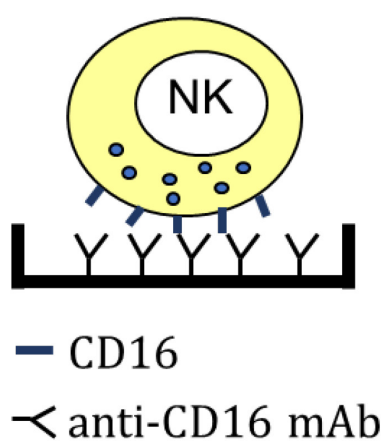

Workflow of stimulating NK cells via CD16 by plate-bound anti-CD16 mAb.

Keywords: Human NK cell, ADCC, CD16, Fc receptor, Plate-bound assay

[Background] Immunotherapies for cancer treatment are rapidly emerging, and many studies aim at 
Please cite this article as: Liu, W. (2022). Evaluating Human Natural Killer Cells Antibody-dependent Cellular Cytotoxicity (ADCC) Using Plate-bound

increasing the safety and efficacy of anti-tumor immunotherapies. More research is being conducted on natural killer (NK) cells, as they have minimal risk of causing neurotoxicity in autologous settings (Gong et al., 2021), graft-versus-host disease in allogenic settings (Du et al., 2021), or cytokine release syndrome in general (Du et al., 2021). Other than being relatively safe in treatment, NK cells are effective in direct killing of target cells and regulating the immune system (Vivier et al., 2011).

Antibody-dependent cellular cytotoxicity (ADCC) is an adaptive immune response largely mediated by NK cells through their CD16 (FCyRIII) receptor, which binds the Fc portion of IgG antibodies (Alderson and Sondel, 2011). However, this important aspect of NK cells remains underexplored. This can be ascribed to difficulties in establishing assay platforms. Previous protocols of ADCC assays use complex cell-based antigen-antibody models, such as the combination of SCC-4 cells and cetuximab (Kohrt et al., 2014), which is costly due to the low productivity of clinically relevant monoclonal antibodies, such as cetuximab. These target cell-based experiments also take a minimum of three days due to the need of culturing and maintaining target cells. The use of anti-CD16 antibody-opsonized P815 cells in reverse ADCC assays also evaluates NK cells ADCC (Euchner et al., 2021), but likewise requires time to culture and maintain the target cells. This current protocol is devoid of target cells, and uses platebound immobilized anti-CD16 antibodies as the trigger. Major steps include coating the plate with CD16 antibodies, preparing, and seeding the cells. It greatly shortens the experimental time to about $10 \mathrm{~h}$, while faithfully evaluating NK cells ADCC. It can be used to quickly evaluate the ADCC aspect of any NK cell-based therapy product in vitro before subsequent in vivo testing, to facilitate the research and development (R\&D) processes.

\section{Materials and Reagents}

1. $1.5 \mathrm{~mL}$ Eppendorf (EP) tube (Eppendorf, catalog number: 022363204)

2. $50 \mathrm{~mL}$ reagent reservoir (Corning, catalog number: CLS4870)

3. $200 \mu \mathrm{L}$ multi-channel pipette (Gilson, catalog number: FA10012)

4. Wide Mouthed Polypropylene Standard Spout Wash Bottle (SP SCIENCEWARE, catalog number: 46C798)

5. Paper towel (Skilcraft, catalog number: 5LA76)

6. Costar 96 well flat-bottom EIA plate (Bio-Rad, catalog number: 2240096)

7. $12 \times 75$ mm FACS tube (BD Biosciences, catalog number: 342065 )

8. DPBS, without calcium chloride and magnesium chloride (Sigma-Aldrich, catalog number: D8537)

9. RPMI medium 1640 (Sigma-Aldrich, catalog number: R8758-24X500ML)

10. Fetal bovine serum (Fisher Scientific, catalog number: 16140089)

11. Penicillin-Streptomycin (Gibco, catalog number: 15-140-122)

12. L-glutamine (Thermo Scientific, catalog number: 25030081)

13. Antibodies:

Ultra-LEAF ${ }^{\mathrm{TM}}$ Purified anti-human CD16 (Bioledgend, catalog number: 302050) 
Anti-CD3-AF700 (BD Biosciences, catalog number: 557943)

Anti-CD14-APC/Cyanine7 (BioLegend, catalog number: 301820)

Anti-CD19-APC/Cyanine7 (BioLegend, catalog number: 302218)

Anti-CD56-PE/Cyanine7 (Beckman Coulter, catalog number: A51078)

Anti-CD107a-BV786 (BD Biosciences, catalog number: 563869)

Anti-IFN-gamma-APC (BioLegend, catalog number: 502512)

Anti-TNF-alpha-Pacific Blue (BioLegend, catalog number: 502920)

14. LIVE/DEADTM Fixable Far Red Dead Cell Stain Kit, for 633 or $635 \mathrm{~nm}$ excitation (Invitrogen, catalog number: L34973)

15. Brefeldin A Solution (1,000x) (BioLegend, catalog number: 420601)

16. BSA (Sigma-Aldrich, catalog number: $12133 \mathrm{C}$ )

17. Sodium Azide (Sigma-Aldrich, catalog number: 71289)

18. Formaldehyde (Sigma-Aldrich, catalog number: F8775)

19. Saponin (Sigma-Aldrich, catalog number: 47036-250G-F)

20. R10 medium (see Recipes)

21. FACS Buffer (see Recipes)

22. Fixation buffer (see Recipes)

23. Perm buffer (see Recipes)

\section{Equipment}

1. $37^{\circ} \mathrm{C} 5 \% \mathrm{CO}_{2}$ humidified incubator (Being Instrument)

2. Countess 3 Automated Cell Counter (Thermo Fisher Scientific)

3. LSR Fortessa flow cytometer (BD Biosciences)

\section{Software}

1. FlowJo Software (version 10.6.1)

\section{Procedure}

A. Plate coating

1. Dilute anti-CD16 antibodies with DPBS, to achieve a final concentration of $2 \mu \mathrm{g} / \mathrm{mL}$. Use $100 \mu \mathrm{L}$ of DPBS for each sample. (The antibody is bottled at the concentration indicated on the vial, typically between $2 \mathrm{mg} / \mathrm{mL}$ and $3 \mathrm{mg} / \mathrm{mL}$. Older lots may have also been bottled at $1 \mathrm{mg} / \mathrm{mL}$. Please contact Biolegend technical support for concentration and total $\mu \mathrm{g}$ amount, or use Biolegend Lookup tool (https://www.biolegend.com/en-us/concentration-expiration-lookup) with the lot number.) 
2. Invert/briefly vortex the diluted anti-CD16 antibodies to mix well and transfer them into a reagent reservoir.

3. Use a $200 \mu \mathrm{L}$ multi-chanel pipette to distribute $100 \mu \mathrm{L}$ of diluted anti-CD16 antibodies into each well of a 96-well EIA plate (the number of wells to be filled depends on the number of NK cell samples to be stimulated). Fill an equal number of wells with DPBS as the no stimulation controls.

4. Cover the plate with a lid and incubate the plate in a $37^{\circ} \mathrm{C} 5 \% \mathrm{CO}_{2}$ humidified incubator for $90 \mathrm{~min}$.

5. Wash off unbound antibodies. Wash the plate twice by first filling the wells with DPBS using a spout wash bottle and then dumping the liquid from the plate. To dump the liquid, grab the plate from the bottom with the thumb in the middle of one side and the fingers on the other side. Holding the plate over the sink, turn the plate upside down and rapidly accelerate arm and hand downward. Immediately place the plate onto paper towels to blot the plate.

6. If NK cells are ready to be seeded (done with Procedure B), continue to Procedure C immediately. Make sure that wells coated with antibodies are not dried for more than $10 \mathrm{~s}$ at any given step. If NK cells are not ready yet, fill wells with $100 \mu \mathrm{L}$ DPBS, cover the plate and store it in the $37^{\circ} \mathrm{C} 5 \mathrm{CO}_{2}$ humidified incubator until cells are ready.

B. NK cell preparation

NK cells for stimulation can be either amidst freshly prepared PBMCs (fresh NK cells) or isolated and expanded in advance (expanded NK cells).

For fresh NK cells:

1. Obtain whole blood upon Institutional Review Board (IRB) approval.

2. Based on the total number of samples, prepare a master mix containing R10 medium, antiCD107a-BV786 antibodies and BFA (v:v:v = 100:2:0.1). The total volume needed for each sample is $200 \mu \mathrm{L}+4 \mu \mathrm{L}+0.2 \mu \mathrm{L}=204.2 \mu \mathrm{L}$.

3. Follow any PBMC isolation protocol to isolate PBMC samples, e.g., EasySep ${ }^{\mathrm{TM}}$ Direct PBMC isolation by STEMCELL Technologies (https://www.stemcell.com/isolating-mononuclear-cellsfrom-whole-blood-by-density-gradient-centrifugation.html).

4. Count the number of cells in each PBMC sample using the Countess 3 Automated Cell Counter.

5. From each PBMC sample, transfer $0.4 \times 10^{6}$ cells into an EP tube.

6. Pellet PBMCs using a benchtop microcentrifuge by centrifuging at $150 \times g$ for $5 \mathrm{~min}$.

7. Resuspend the pelleted $0.4 \times 10^{6}$ cells in each EP tube with $204.2 \mu \mathrm{L}$ of master mix. (BFA is cytotoxic, so conduct step 7 only close to the end of the $90 \mathrm{~min}$ incubation period in Procedure A step 4. Do not collect more than $0.4 \times 10^{6}$ cells/EP tube. The cells will be seeded into a well coated with CD16 antibodies. Too many cells will prohibit cell-antibody interaction.)

For expanded NK cells (an example of FcRy KO cells are shown, Figure 1): 
1. Count the number of cells in each sample using the Countess 3 Automated Cell Counter.

2. Based on the total number of samples, prepare a master mix containing R10 medium, antiCD107a-BV786 antibodies and BFA (v:v:v = 100:2:0.1). The total volume needed for each sample is $200 \mu \mathrm{L}+4 \mu \mathrm{L}+0.2 \mu \mathrm{L}=204.2 \mu \mathrm{L}$.

3. From each sample, transfer $0.02 \times 10^{6}$ cells into an EP tube.

4. Pellet cells using a benchtop microcentrifuge at $800 \times g$ for $5 \mathrm{~min}$.

5. Resuspend cells using an appropriate amount of R10 medium (This step washes off stimulatory components in NK cell culture and will rest the cells).

6. Pellet cells using a benchtop microcentrifuge at $150 \times g$ for $5 \mathrm{~min}$.

7. Resuspend the pelleted $0.02 \times 10^{6}$ cells in each EP tube with $204.2 \mu \mathrm{L}$ master mix. (BFA is cytotoxic, so conduct step 7 only close to the end of the 90 min incubation period in Procedure A step 4).

C. NK cell stimulation

1. For each sample, seed $100 \mu \mathrm{L}$ of cells into one well coated with anti-CD16 antibodies and another $100 \mu \mathrm{L}$ of cells into one well coated with no anti-CD16 antibody.

2. Cover the plate and incubate the cells in a $37^{\circ} \mathrm{C} 5 \% \mathrm{CO}_{2}$ humidified incubator for $6-9 \mathrm{~h}$.

D. Flow cytometric analysis

1. Before collecting the cells, prepare the surface staining cocktail (needs to be protected from light). Prepare a master mix using the following guideline: For each sample to be stained, use $50 \mu \mathrm{L}$ of FACS buffer containing $0.2 \mu \mathrm{L}$ of LIVE/DEAD ${ }^{\text {TM }}$ Fixable Far Red Dead Cell Stain, $1 \mu \mathrm{L}$ of Anti-CD3-AF700, $1 \mu \mathrm{L}$ of Anti-CD14-APC/Cyanine7, $1 \mu \mathrm{L}$ of Anti-CD19-APC/Cyanine7, and $1 \mu \mathrm{L}$ of Anti-CD56-PE/Cyanine7 antibodies. (If staining with a different volume, use manufacturers' suggested concentrations.)

2. Place the plate on ice to slow down cellular activities.

3. Add $100 \mu \mathrm{L}$ of ice-cold FACS buffer into each well that has cells seeded.

4. Transfer cells into FACS tubes. Pipet up and down for multiple times to make sure that all cells are off the plate.

5. Pellet the cells by centrifuging at $350 \times g$ for $5 \mathrm{~min}$.

6. Briefly vortex to dislodge the cells.

7. Add $54.2 \mu \mathrm{L}$ surface antibody cocktail into each cell suspension, and stain on ice for $30 \mathrm{~min}$.

Note: Protect cells from light from this step onwards.

8. Wash cells twice with $3 \mathrm{~mL}$ of ice-cold FACS buffer at $350 \times \mathrm{g}$ for $5 \mathrm{~min}$ and discard supernatant.

9. Briefly vortex to dislodge the cells.

10. Fix cells with fixation buffer (working concentration: $1 \% \mathrm{v} / \mathrm{v}$ formaldehyde) on ice for $30 \mathrm{~min}$.

11. Permeabilize cells by adding $3 \times$ volume of ice-cold Perm buffer to fixed cells.

12. Keep cells on ice for $30 \mathrm{~min}$. 
13. Prepare intracellular staining cocktail. Prepare a master mix using the following guideline: For each sample to be stained, use $50 \mu \mathrm{L}$ of Perm buffer containing $2.5 \mu \mathrm{L}$ of Anti-IFN-gamma-APC and $2.5 \mu \mathrm{L}$ of Anti-TNF-alpha-Pacific Blue antibodies. (If staining with a different volume, use manufacturers' suggested concentrations.)

14. Wash cells twice with $3 \mathrm{~mL}$ of ice-cold Perm buffer at $600 \times g$ for $5 \mathrm{~min}$ and discard supernatant.

15. Briefly vortex to dislodge the cells.

16. Add $55 \mu \mathrm{L}$ of intracellular antibody cocktail into each cell suspension and stain at RT for $30 \mathrm{~min}$.

17. Wash cells twice with $3 \mathrm{~mL}$ of ice-cold Perm buffer at $600 \times g$ for $5 \mathrm{~min}$ and discard supernatant.

18. Wash cells once with $3 \mathrm{~mL}$ of ice-cold FACS buffer at $600 \times g$ for $5 \mathrm{~min}$ and discard supernatant.

19. Acquire cells by LSR Fortessa flow cytometer.

20. Analyze the data using FlowJo Software.

\section{Data analysis}

An example of stimulating expanded NK cells is shown here (Figure 1). NK cells from different blood donors are first expanded and then electroporated with FCER1G-targeting CRISPR-Cas9 ribonucleoprotein (FCER1G encodes for FcRy). The resultant cells are a mixture of $\mathrm{FcRY}^{+}$and FcRY- cells. During stimulation with plate-bound anti-CD16 antibodies, the cells are stained for surface CD107a (a degranulating marker). After stimulation, the cells are first stained for surface markers and then stained for intracellular cytokines (IFN- $\gamma$ and TNF- $\alpha$ ). In analysing data using FlowJo Software, cells are gated by FSC-A/SSC-A to exclude debris and by FSC-H/FSC-A to select

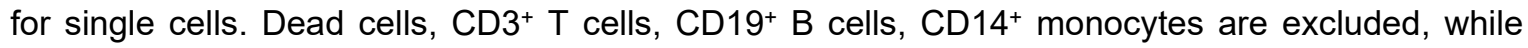
$\mathrm{CD} 56^{+} \mathrm{NK}$ cells are selected for intracellular cytokines and degranulation marker analysis. 
A

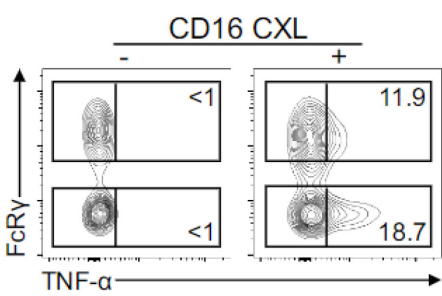

B

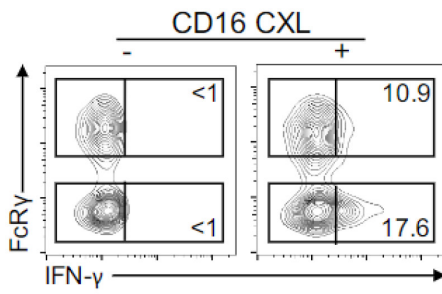

C

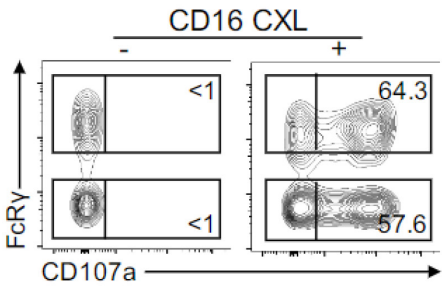

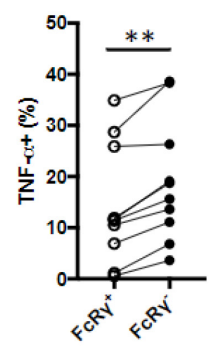
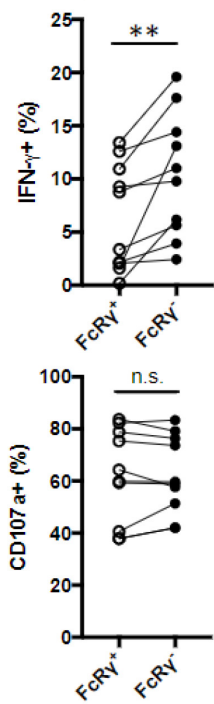

Figure 1. Deletion of FCER1G (encoding FcRY) in expanded NK cells leads to enhanced ADCC (exemplified by cytotoxic cytokine release).

(A-C) Contourplots show flow cytometric analysis of TNF- $\alpha$ (A), IFN- $\gamma$ production (B) and cell surface $\mathrm{CD}$ 107a expression (C) in $\mathrm{FcRY}^{+}$and $\mathrm{FCRY}^{-} \mathrm{NK}$ subsets from a representative donor without (-) or with (+) CD16 crosslinking (CXL). Numbers represent the relative percentages of $\mathrm{FcRY}^{+}$or FcRY- subset that produced the indicated cytokine or displayed CD107a. Line graphs show the relative percentages of $\mathrm{FcRY}^{+}$or $\mathrm{FcRY}^{-}$subset that produced the indicated cytokine or displayed CD107a from several donors in response to CD16 CXL $(n=10)$. Circles connected by a line designate data obtained from the same donor sample.

\section{$\underline{\text { Recipes }}$}

1. R10 medium

RPMI medium 1640 supplemented with $10 \%$ fetal bovine serum. $100 \mathrm{U} / \mathrm{mL}$ Penicillin-Streptomycin and $2 \mathrm{mM}$ L-glutamine.

2. FACS Buffer PBS supplemented with $1 \%$ BSA and $0.1 \%$ Sodium Azide.

3. Fixation buffer PBS supplemented with $4 \%$ formaldehyde.

4. Perm buffer

PBS supplemented with $1 \%$ BSA and $0.1 \%$ saponin. 


\section{Acknowledgments}

This protocol is adapted from Liu et al. (2020). The work of Liu et al. (2020) was supported by the NIH grant ( Al110894).

\section{Competing interests}

The authors declare no competing interests.

\section{Ethics}

Studies exemplified in this protocol using human samples were approved by the Institutional Review Board of the University of California, Davis.

\section{References}

1. Alderson, K. L. and Sondel, P. M. (2011). Clinical cancer therapy by NK cells via antibodydependent cell-mediated cytotoxicity. J Biomed Biotechnol 2011: 379123.

2. Du, N., Guo, F., Wang, Y. and Cui, J. (2021). NK Cell Therapy: A Rising Star in Cancer Treatment. Cancers (Basel) 13(16).

3. Euchner, J., Sprissler, J., Cathomen, T., Furst, D., Schrezenmeier, H., Debatin, K. M., Schwarz, K. and Felgentreff, K. (2021). Natural Killer Cells Generated From Human Induced Pluripotent Stem Cells Mature to CD56 bright $\mathrm{CD} 16^{+} \mathrm{NKp80^{+- }}$ In-Vitro and Express KIR2DL2/DL3 and KIR3DL1. Front Immunol 12: 640672.

4. Gong, Y., Klein Wolterink, R. G. J., Wang, J., Bos, G. M. J. and Germeraad, W. T. V. (2021). Chimeric antigen receptor natural killer (CAR-NK) cell design and engineering for cancer therapy. J Hematol Oncol 14(1): 73.

5. Kohrt, H. E., Colevas, A. D., Houot, R., Weiskopf, K., Goldstein, M. J., Lund, P., Mueller, A., Sagiv-Barfi, I., Marabelle, A., Lira, R., et al. (2014). Targeting CD137 enhances the efficacy of cetuximab. J Clin Invest 124(6): 2668-2682.

6. Liu, W., Scott, J. M., Langguth, E., Chang, H., Park, P. H. and Kim, S. (2020). FcRgamma Gene Editing Reprograms Conventional NK Cells to Display Key Features of Adaptive Human NK Cells. iScience 23(11): 101709.

7. Vivier, E., Raulet, D. H., Moretta, A., Caligiuri, M. A., Zitvogel, L., Lanier, L. L., Yokoyama, W. M. and Ugolini, S. (2011). Innate or adaptive immunity? The example of natural killer cells. Science 331(6013): 44-49. 